Revue internationale P.M.E.

Économie et gestion de la petite et moyenne entreprise

Revure

internationale

PME

\title{
Le groupe stratégique des entreprises de terroir
}

\section{Jean-Louis Rastoin et Véronique Vissac-Charles}

Volume 12, numéro 1-2, 1999

URI : https://id.erudit.org/iderudit/1008655ar

DOI : https://doi.org/10.7202/1008655ar

Aller au sommaire du numéro

Éditeur(s)

Presses de l’Université du Québec

ISSN

0776-5436 (imprimé)

1918-9699 (numérique)

Découvrir la revue

Citer cet article

Rastoin, J.-L. \& Vissac-Charles, V. (1999). Le groupe stratégique des entreprises de terroir. Revue internationale P.M.E., 12(1-2), 171-192.

https://doi.org/10.7202/1008655ar

\section{Résumé de l'article}

Ce texte a pour objet d'analyser les conditions d'émergence d'un groupe stratégique "PME de terroir» dans le système alimentaire. Le positionnement des produits de terroir par rapport aux autres produits alimentaires est fondé sur la notion de qualité. Les crises récentes qui ont secoué le système alimentaire (ESB, hormones, OGM, etc.) font resurgir l'importance de la confiance accordée par le consommateur à un produit lors de l'acte d'achat. Cette confiance peut être liée à une réputation, une histoire, un patrimoine culturel. Dans ce cas, l'identité du produit et l'identité de l'entreprise sont indissociables. Cependant, malgré des réglementations diverses (AOC, AOP, IGP, etc.), le concept de terroir reste flou. Se pose alors le problème de la valorisation des produits de terroir, alors qu'il existe un vivier de PME capables de se lancer ou d'amplifier leur action sur ce créneau. Les PME, si elles veulent garder leur avantage concurrentiel sur ce type de marché, doivent construire des barrières à l'entrée suffisamment fortes. Elles doivent élaborer une stratégie collective consistant à favoriser et à renforcer les mises en équivalence entre qualité - sécurité - diversité - innovation - aménagement du territoire, ainsi que leurs compétences managériales pour créer de la valeur. 


\title{
Le groupe stratégique des entreprises de terroir
}

\author{
Jean-Louis RASTOIN \\ Véronique VISSAC-CHARLES \\ Agro-Montpellier
}

MOTS CLÉS

PME - Agroalimentaire - Terroir - Groupe stratégique - Qualité Sécurité alimentaire - Marchés - Innovation - Création de valeur

\begin{abstract}
RÉSUMÉ
Ce texte a pour objet d'analyser les conditions d'émergence d'un groupe stratégique «PME de terroir » dans le système alimentaire. Le positionnement des produits de terroir par rapport aux autres produits alimentaires est fondé sur la notion de qualité. Les crises récentes qui ont secoué le système alimentaire (ESB, hormones, OGM, etc.) font resurgir l'importance de la confiance accordée par le consommateur à un produit lors de l'acte d'achat. Cette confiance peut être liée à une réputation, une histoire, un patrimoine culturel. Dans ce cas, l'identité du produit et l'identité de
\end{abstract}

\section{LES AUTEURS}

Jean-Louis Rastoin, ingénieur agronome, détenteur d'un doctorat en sciences économiques et agrégé de sciences de gestion, est actuellement professeur d'économie et gestion des entreprises à l'École nationale supérieure agronomique de Montpellier. Auteur de plus de 80 publications, il mène, dans le cadre du GRAAL-Montpellier, des recherches sur les stratégies d'entreprises dans le système alimentaire français, européen et mondial. Adresse: ENSA/UFR Economie et gestion des entreprises/GRAAL-M, 2, place Pierre-Viala, 34060 Montpellier Cedex 2, France. Téléphone: 33499612589 ou 28 81. Télécopieur : 3346763 54 09. Courriel: rastoin@ensam.inra.fr

Véronique Vissac-Charles, ingénieure en génie rural des eaux et des forêts et détentrice d'un doctorat en socio-économie, est actuellement chercheure à l'École nationale supérieure agronomique de Montpellier. Ses recherches sont centrées sur la gestion des projets d'innovation et sur les formes d'accompagnement de l'innovation que mettent en place les établissements d'enseignement supérieur et de recherche. Adresse: ENSA/UFR Économie et gestion des entreprises/GRAAL-M, 2, place Pierre-Viala, 34060 Montpellier Cedex 2, France. Téléphone: 33499612589 ou 28 81. Télécopieur: 33467635409. Courriel : vissac-charles@ensam.inra.fr 
l'entreprise sont indissociables. Cependant, malgré des réglementations diverses (AOC, AOP, IGP, etc.), le concept de terroir reste flou. Se pose alors le problème de la valorisation des produits de terroir, alors qu'il existe un vivier de PME capables de se lancer ou d'amplifier leur action sur ce créneau. Les PME, si elles veulent garder leur avantage concurrentiel sur ce type de marché, doivent construire des barrières à l'entrée suffisamment fortes. Elles doivent élaborer une stratégie collective consistant à favoriser et à renforcer les mises en équivalence entre qualitésécurité - diversité - innovation - aménagement du territoire, ainsi que leurs compétences managériales pour créer de la valeur.

\section{ABSTRACT}

The aim of this text is to analyze the emergence conditions of a "PME de terroir" strategic group in the agrofood system. The "terroir" products positioning compared to other food products is based on the notion of quality. The recent crisis which have shaken the agrofood system (ESB, hormones, OGM...) reveal the primary role of trust in product buying. Trust may be linked to reputation, history, cultural heritage. In this case product and firm identities are indissociable. In spite of varied legislations (AOC, AOP, IGP...), the "terroir" concept is still vague. "Terroir" product valorisation stays a problem while many SMEs are capable of entering the market or of increasing their action in this opening market. If SMEs want to keep their competitive advantage in this market they must construct appropriate entry barriers. They must elaborate a collective strategy to promote and reinforce the links between quality - security - innovation - regional development, as well as their management skills to create value.

\section{RESUMEN}

El objecto de este papel es de analysar las condiciones de emergencia de un grupo estrategico «PME de terroir » en el sistema alimenticio. El posicionamiento de los productos de «terroir ", en comparación con otros productos alimenticios, se funde en la noción de cualidad. Las recientes crisis que han agitado el sistema alimenticio (ESB, hormonas, OGM ...) enfoquen en la importancia de la confianza que el consumidor concede a un producto cuando le compro. Esta confianza puede ser ligada a una reputación, una historia o un patrimonio cultural. En este caso, la identitad del producto y la identitad de la empresa estan indisociables. Pero, a pesar de variadas reglamantaciónes (AOC, AOP, IGP...), el concepto de « terroir » queda vago. El problema de la valorización de los productos de «terroir " se plantea, cuando existen muchas PyMEs capaces de lanzarse o de ampliar sus acciónes en este mercado. Si quieren guardar su situación competitiva en este tipo de mercado, las PyMEs deben construir barreras de entrada bastante fuertas. Deben elaborar una estrategia colectiva para favorecer y intensificar las equivalencias entre cualidadsecuridad - varidedad-innovación - desarollo regional, así como sus competencias empresariales para crear valor.

\section{ZUSAMMENFASSUNG}

Dieser Text analysiert die Ursache für die Gründung einer strategischen Vereinigung "KMU im Ackerbau» im Nahrungsmittelsystem. Die Positionierung von Ackerbauprodukten ist im Vergleich zu anderen Nahrungsmitteln auf Qualität 
ausgerichtet. Die neuesten Krisen im Nahrungsmittelbereich (BSE, Hormone, u.a.) zeigen auf, welche Wichtigkeit dem Vertrauen des Konsumenten beim Kauf eines Produktes beigemessen werden muss. Dieses Vertrauen kann verbunden sein mit einem guten Ruf, einer Betriebsgeschichte, einem Kulturgut. In diesem Falle sind Identität des Produktes und Identität der Unternehmung unzertrennlich. Trotz diverser Reglementierungen bleibt das Ackerbaukonzept unklar. Eine Vielzahl von $K M U$ sind fähig, diesen Markt zu bearbeiten. Es stellt sich lediglich das Problem, wie die Ackerbauprodukte aufgewertet werden können. Wollen die KMU ihren Konkurrenzvorteil auf diesem Markt behalten, müssen sie genügend hohe Eintrittsbarrieren schaffen. Sie müssen eine kollektive Strategie ausarbeiten, welche das Gleichgewicht zwischen Qualität, Sicherheit, Vielfalt, Innovation, Raumplanung fördert und verstärkt, und sie müssen ihre Managementfähigkeiten verbessern, um die Wertschöpfung sicherzustellen.

\section{Introduction}

Le terme d'entreprise de terroir a été vulgarisé depuis quelques années ${ }^{1}$, à la suite des mutations observées dans les modèles de consommation des ménages, avec le nouvel intérêt porté aux produits dits «naturels ». Nous retiendrons la définition suivante : une entreprise de terroir est une entreprise qui tire sa spécificité de liens de forte intensité avec un territoire identifié par des caractéristiques physiques (géographiques et agroclimatiques), historiques et sociales, c'est-à-dire culturelles. Les exemples d'entreprise de terroir se trouveront en conséquence en grande majorité dans le secteur agroalimentaire. On peut cependant considérer que les entreprises du secteur de l'artisanat non alimentaire à fort contenu culturel (on peut citer ici les métiers de l'art traditionnel) relèvent également de cette catégorie.

Les fondements théoriques du concept de groupe stratégique des « entreprises de terroir » participent de deux courants fondamentaux :

- l'analyse stratégique, formalisée dans le cadre de l'économie industrielle, que l'on peut rattacher aux sciences de gestion;

- l'économie spatiale, hybride des sciences économiques et géographiques.

Michael Porter (1980) $)^{2}$ est l'un des premiers à avoir défini, au début des années 1980, la notion de groupe stratégique. Pour cet auteur, il s'agit d'une population d'entreprises adoptant un comportement stratégique commun au sein d'un secteur de référence. Ce secteur sera ici le «système alimentaire ». Un système alimentaire (Rastoin, 1995) est un réseau interdépendant d'acteurs (entreprises,

1. Nous l'avons utilisé, pour notre part, dans un ouvrage consacré aux politiques agricoles, cf. Rastoin (1992).

2. Porter (1982), p. 142-170. 
institutions financières, organismes publics et privés), localisé dans un espace géographique donné (région, État, espace plurinational) et participant directement ou indirectement à la création de flux de biens et services orientés vers la satisfaction des besoins alimentaires de un ou plusieurs groupes de consommateurs localement ou à l'extérieur de la zone considérée. Une représentation modélisée du système alimentaire français, construite à partir du tableau «entrées-sorties » de la comptabilité nationale, selon une logique technique, permet de présenter les différents opérateurs / processeurs du système :

- agrofourniture et agroéquipements ;

- agriculture ;

- agro-industries de transformation des matières premières agricoles (IAA);

- appareil de distribution des biens alimentaires (distribution et restauration);

- activités d'appui (industries d'équipements et de produits intermédiaires, services, administrations, formation, R-D).

La théorie des systèmes permet de prendre en considération la finalité de l'activité agroalimentaire, les interrelations entre les agents de toute nature (y compris par exemple les nutritionnistes-prescripteurs, les médias, les associations de consommateurs, les pouvoirs publics nationaux et supranationaux, etc.), la structure du système alimentaire caractérisée par des variables d'état (la concentration des entreprises à un instant $t$, par exemple), des variables imposées (les taux d'intérêt directeurs dans le pays considéré) et des variables d'action (le niveau d'emploi dans le système).

Le nombre et la diversité des acteurs rencontrés au sein du système alimentaire sont extrêmes puisque cohabitent, dans un pays comme la France, quelques dizaines de très grandes firmes multinationales (dans l'agrofourniture, l'industrie alimentaire et la distribution), quelques milliers de PME et des centaines de milliers de microentreprises (essentiellement dans l'agriculture, la transformation et le commerce de détail).

L'identification des groupes stratégiques au sens de Porter renvoie à un autre concept de l'auteur, celui « d'avantage concurrentiel » (Porter, 1990). Porter postule qu'il existe deux grands types d'avantages concurrentiels :

- la domination par les coûts ;

- la différenciation.

Un avantage par les coûts signifie que l'entreprise est capable de produire en dépensant moins par unité. De multiples possibilités de réduction des coûts existent depuis la conception jusqu'à la commercialisation d'un produit et doivent être systématiquement recherchées par les entreprises. En tout état de cause, 
l'utilisation des économies d'échelle dans le processus de fabrication reste le moyen le plus sûr d'abaisser significativement les coûts dans l'activité agroalimentaire.

La différenciation est la capacité à fournir à l'acheteur un produit qu'il va considérer comme unique et supérieur aux autres au regard de un ou plusieurs de ses attributs. Elle peut être obtenue soit par des techniques de marketing (par exemple, la segmentation des clientèles et la construction d'une image de marque, un type de stratégie utilisé massivement par les très grandes firmes multinationales [TGFM], qui en ont les moyens financiers), soit par une forte «caractérisation » du produit (on s'affranchit alors de la «dictature » du chiffre d'affaires, puisque les entreprises de taille réduite peuvent adopter une telle stratégie).

$$
\text { TABleau } 1
$$

\section{Les « stratégies de base » de Porter appliquées au système alimentaire}

\begin{tabular}{|c|c|c|}
\hline \multirow[b]{3}{*}{$\begin{array}{l}\text { Cible } \\
\text { large }\end{array}$} & \multicolumn{2}{|c|}{ Avantage concurrentiel } \\
\hline & Coûts & Différenciation \\
\hline & $\begin{array}{l}\text { 1. Domination } \\
\text { par les coûts : } \\
\text { production de masse }\end{array}$ & $\begin{array}{l}\text { 3. Segmentation } \\
\text { «marketing» }\end{array}$ \\
\hline $\begin{array}{l}\text { Cible } \\
\text { étroite }\end{array}$ & $\begin{array}{l}\text { 2. Stratégie de coûts : } \\
\text { implantation }\end{array}$ & $\begin{array}{l}\text { Différenciation } \\
\text { «spécialisée» } \\
\text { 4. Produits innovants } \\
\text { 5. Produits du terroir }\end{array}$ \\
\hline
\end{tabular}

Source : Adapté de Porter (1985).

Dans les deux cas, l'avantage concurrentiel se manifestera à travers une productivité plus grande des ressources engagées, soit par la baisse du dénominateur (coût des facteurs), soit par la hausse du numérateur (prix).

En considérant que ces avantages peuvent être exercés sur des marchés (champs concurrentiels) larges ou, au contraire, étroits, Porter construit une matrice stratégique à quatre cases, devenue un classique de l'analyse stratégique. Une lecture du concept de système alimentaire à travers la grille portérienne conduit à repérer cinq types de positionnement stratégique (on relèvera au passage que le paradigme des quatre cases est ici aménagé) :

1. coûts minimisés par une production de masse;

2. coûts réduits par impartition, sur des volumes faibles; 
3. différenciation-produit "périphérique » (modelage du goût par des ingrédients et construction d'image par l'emballage et la communication), sur la base d'une segmentation fine du marché ;

4. différenciation par l'innovation-produit;

5. différenciation par le couple territoire - histoire.

\section{La résistible ascension des produits de terroir}

L'application du concept portérien d'avantages concurrentiels au système alimentaire conduit donc à envisager quatre groupes stratégiques, par référence aux cinq types de marchés qui viennent d'être présentés. Deux groupes stratégiques empruntent au principe de domination par les coûts et la segmentation et les deux autres, au principe de différenciation «spécialisée » :

- le groupe stratégique des TGFM agroalimentaires sur marchés de masse standard (1);

- le groupe stratégique des PME d'impartition sur marchés standard (2);

- le groupe stratégique des TGFM agroalimentaires et pharmaceutiques sur marchés innovants (3);

- le groupe stratégique des entreprises de terroir (4).

Le premier groupe stratégique (que nous intitulerons « $\mathrm{CS}$ », pour domination par les coûts et la segmentation) est de loin le plus important puisqu'il occupe environ $90 \%$ du marché des produits alimentaires dans les pays à hauts revenus. Il concerne des produits alimentaires «basiques », c'est-à-dire de qualité standard, respectant évidemment les normes de santé publique, mais dont la variable déterminante d'achat par le consommateur reste le prix. Ce phénomène est amplifié, voire provoqué, par le pouvoir de marché exercé par les grands distributeurs (en France, les trois quarts des produits alimentaires sont aujourd'hui vendus par les GMS ${ }^{3}$ ). En conséquence, les firmes intervenant sur ce marché sont conduites à minimiser leurs coûts par une production de masse et à segmenter leurs clientèles pour répondre à une pression concurrentielle de plus en plus vive. La recherche de la taille critique internationale est ici un facteur déterminant de la stratégie des firmes agroalimentaires qui doivent, pour assurer leur survie compte tenu d'une pression concurrentielle vigoureuse, consentir des investissements matériels et immatériels (essentiellement en communication) considérables. Cela a conduit à la constitution d'un oligopole d'une centaine de firmes dont la taille moyenne équivalait, en 1996, à 9 milliards de dollars américains de chiffre d'affaires annuel, 40000 salariés et une implantation dans 15 pays.

3. GMS : grandes et moyennes surfaces. 
FIGURE 1

Carte des groupes stratégiques agroalimentaires à la fin des années 1990

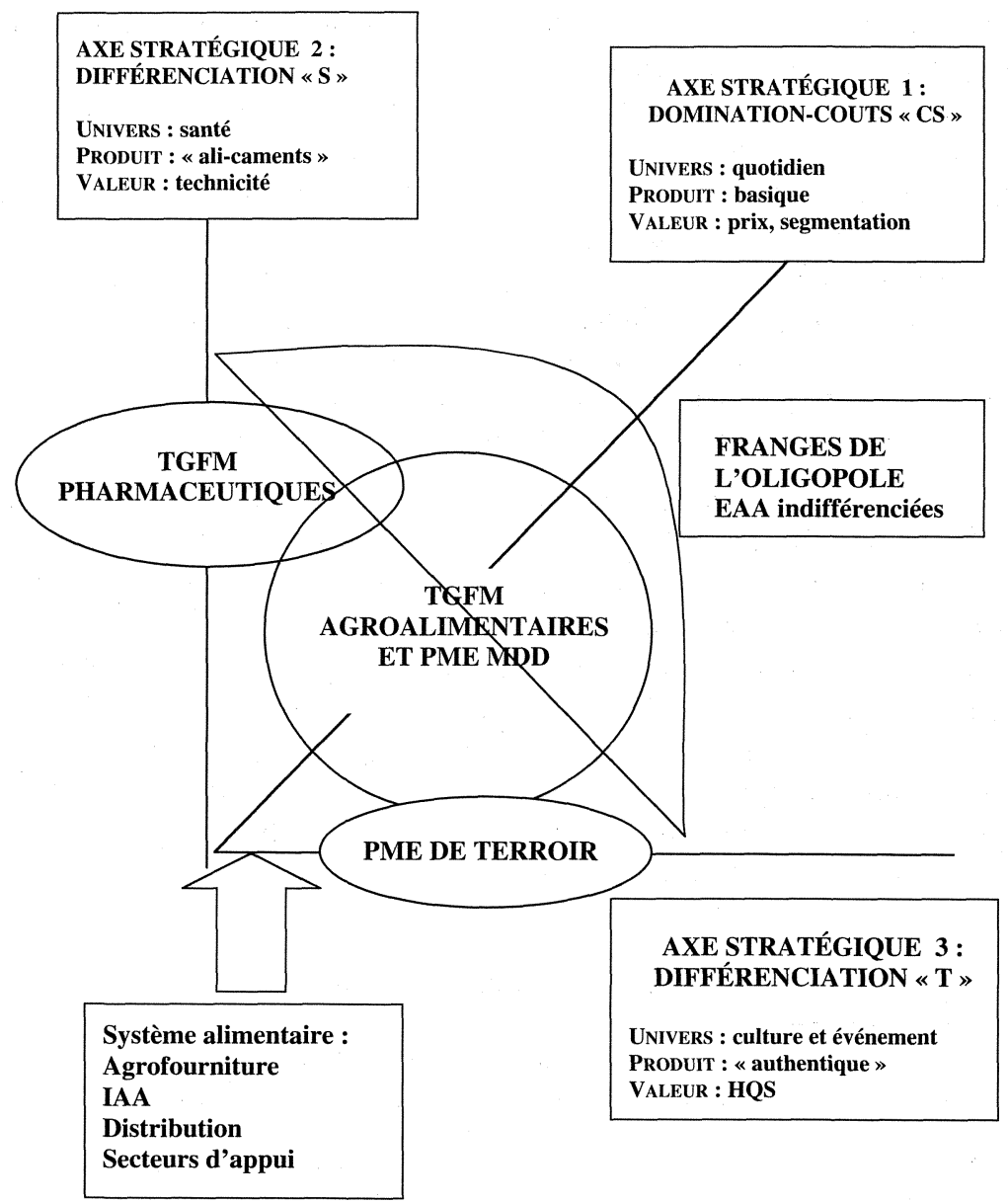

La sous-traitance constitue le deuxième moyen de réduire les charges de l'entreprise en éliminant les coûts marketing et une partie des frais de R-D qui sont alors pris en charge par le donneur d'ordres : c'est la solution adoptée par les entreprises qui acceptent un partenariat-aval pour la fabrication de produits sous marque de distributeur (MDD). Dans ce cas, la PME devient compétitive. La volonté d'autonomisation des distributeurs par rapport aux géants industriels et le bénéfice d'image retiré d'une coopération affichée avec des entreprises régionales laissent un espace à un certain nombre de PME acceptant (ou recherchant) la position de sous-traitant. 
Le troisième groupe (différenciation « $S$ », pour santé) concerne le marché en croissance rapide des « ali-caments » (aliments-médicaments destinés à des groupes particuliers de consommateurs, par exemple, bébés, femmes enceintes, seniors, sportifs, mais aussi consommateurs en situation pathologique : obèses, diabétiques, séropositifs, etc.). Initialement élaborés par les firmes pharmaceutiques, ces produits sont de plus en plus intégrés dans les domaines d'activité stratégique des groupes alimentaires. Ainsi, Danone, à la suite de la cession de ses actifs du secteur des pâtes et de l'épicerie, est en cours de réorganisation en trois grandes divisions : produits santé, aliments de grignotage (snack-foods) et boisson. On peut également citer les lignes de produits «weight watchers » de H.J. Heinz, «health's choice » de Conagra et les aliments cliniques des filiales BioItalia et Euro-Aspartame de la firme japonaise Ajinomoto. Les «ali-caments » sont fortement différenciés par une image «hygiène et santé », domaine dans lequel le consommateur ne se détermine pas en fonction du prix de vente, mais de «l'utilité » et de la technicité du produit.

Le quatrième groupe (différenciation « $T$ », pour terroir), enfin, relève d'une stratégie de différenciation de type «culturel». Il concerne les produits alimentaires à forte identité (PAFI). En effet, à côté de la consommation d'aliments standard « quotidiens », qui représente aujourd'hui encore quantitativement l'essentiel de la consommation totale, se développe un marché de l'aliment différencié par des caractères distinctifs identifiables ${ }^{4}$ : les paramètres organoleptiques, les étapes de production, l'histoire sociale et le territoire d'origine. À partir de ces éléments, il devient possible de construire une typologie des produits alimentaires ${ }^{5}$ en distinguant ce que l'on pourrait appeler les produits de terroir, des produits innovants et des produits basiques.

TABLEAU 2

Typologie des produits alimentaires

\begin{tabular}{lccccc}
\hline $\begin{array}{l}\text { Type de } \\
\text { produits }\end{array}$ & $\begin{array}{c}\text { Caractéristiques } \\
\text { organoleptiques }\end{array}$ & $\begin{array}{c}\text { Origine } \\
\text { territoriale }\end{array}$ & $\begin{array}{c}\text { Procédé de } \\
\text { production }\end{array}$ & $\begin{array}{c}\text { Histoire } \\
\text { sociale }\end{array}$ & Prix \\
\hline de terroir & très typées & $\begin{array}{c}\text { clairement } \\
\text { spécifiée }\end{array}$ & $\begin{array}{c}\text { artisanal } \\
\text { «normalisé }\end{array}$ & très présente & élevé \\
\hline innovants & $\begin{array}{c}\text { relativement } \\
\text { neutres }\end{array}$ & non nécessaire & $\begin{array}{c}\text { technologie } \\
\text { avancée }\end{array}$ & inexistante & très élevé \\
\hline basiques & segmentées & diversifiée & de masse & $\begin{array}{c}\text { parfois évoquée } \\
\text { (marketing) }\end{array}$ & faible \\
\hline
\end{tabular}

4. Sur la notion de qualité alimentaire et de signes distinctifs des produits, voir les travaux de Sylvander (1995).

5. Cette typologie fait appel aux concepts développés par Malassis et Ghersi (1996). 
Les produits de terroir sont fortement ancrés dans une histoire, une culture, une aire géographique. Leur valeur «intrinsèque », à la fois matérielle (qualités organoleptiques) et subjective / évocative ou symbolique est forte, à tel point que le prix ne constitue pas la variable déterminante d'achat. Les produits innovants tirent leur qualité spécifique d'une technologie et d'une valeur ajoutée qui peut être un service (facilité de préparation) ou un avantage nutritionnel (aliment santé). Pour eux aussi, le prix peut être élevé, procurant des marges substantielles qui permettent au pionnier de bénéficier de la rente d'innovation pendant un certain temps. Les produits basiques sont caractérisés par leur production et leur distribution de masse, par des prix très concurrentiels, par une segmentation marketing poussée et par l'utilisation systématique de l'effet de notoriété de la marque.

Les différents chocs qu'a subis le système alimentaire - intoxications alimentaires dues à la mauvaise maitrise de certaines bactéries (Listeria, Salmonella), incertitude sur l'encéphalopathite spongiforme bovine (ESB) et sa transmission à l'homme, problème des hormones dans la viande, des pesticides dans les fruits et légumes, de la dégradation du goût des produits, organismes génétiquement modifiés (OGM) - ont incité les consommateurs à douter de l'innocuité des produits dont ils se nourrissent. La méfiance s'est progressivement installée. L'incertitude devient une composante importante du marché de l'agroalimentaire, le progressif refus de l'opacité oblige à rouvrir les boîtes noires des pratiques de production et de transformation. Dès qu'il existe une indétermination sur la nature des produits, des problèmes de coordination émergent (Holmstrom et Tirole, 1989). Reconstruire la confiance devient alors un des enjeux fondamentaux du maintien de l'acte marchand. Pour cela, il faut redéfinir les repères collectifs autour desquels producteurs et consommateurs vont s'articuler (Thévenot, 1986) et reconstruire les conventions permettant de structurer l'espace commun (Eymard-Duvernay, 1994). Après une déstabilisation des réseaux traditionnels, les relations entre les différents acteurs vont se réorganiser, favorisant une dynamique de transformation de ces réseaux autour de nouveaux produits (Vissac-Charles, 1996). L'objectivation des produits passe par une instrumentation, une intégration de nouveaux programmes d'actions dans des dispositifs techniques (Akrich, Callon et Latour, 1988) permettant aussi la mise au point de nouvelles procédures (la traçabilité des produits, par exemple) participant fortement au renouvellement des réseaux.

Le besoin de sécurité exprimé par le consommateur oblige les IAA à ne plus se comporter comme des vendeurs anonymes sur le marché, le jugement sur la transaction passant au préalable par un jugement sur l'entreprise. La réputation devient l'un des déterminants de l'acte d'achat. Mais la réputation suppose une capitalisation temporelle. Les nouvelles entreprises entrant sur le marché ne peuvent donc s'appuyer que sur l'image associée à une région, à une façon de produire, à un terroir (Sylvander, 1995). En liant sa spécificité aux caractéristiques du 


\section{produit, l'entreprise fait émerger de nouvelles règles de production, de trans- formation et de distribution des produits.}

$\mathrm{Au}$ fond, on pourrait dire que si le terroir ne garantit pas l'innocuité des produits, il y a néanmoins deux façons, non exclusives, de construire la confiance :

- objectivement, par les normes (l'État, la puissance publique) et la capacité à les respecter, ce qui semble plus accessible aux grandes firmes qu' aux petites en raison des coûts élevés de transaction (procédures longues de certification);

- subjectivement, par l'histoire, la tradition, la réputation, garantie par la «terre des ancêtres », et le territoire. Cette deuxième approche correspond à la problématique «PME de terroir » et permet en outre de passer de la qualité-sécurité à la qualité-goût et au caractère «unique», donc inimitable du produit.

Cependant, le produit n'est que l'un des éléments permettant de définir un groupe stratégique. Il importe également de considérer la nature de la firme et son comportement. Dans le cas du groupe «terroir», la dimension de l'entreprise doit être proportionnée à l'espace géographique de production et reste donc limitée (PME/TPE). Les comportements seront alors plus dictés par des logiques entrepreneuriales que patrimoniales, au sens de Marchesnay (1995).

\section{Le maquis de l'identification des « produits de terroir »}

La construction d'une identité « terroir» peut, dans certains cas, reposer sur des normes et des réglementations (AOC, IGP, AOP). Dans ce cas, les procédures auxquelles l'entreprise doit se conformer sont claires et bien identifiées. Au-delà de ces aspects réglementaires, qui décrivent des réseaux fortement stabilisés, il faut essayer d'analyser la construction conjointe de l'identité d'une entreprise et de ses produits, repérer les éléments du réseau qui participent à la construction de l'identité et comprendre de quelle manière cela s'inscrit dans des dispositifs techniques, des savoir-faire, des normes de production réglementaires ou non.

Les principales qualités dont doivent être pourvues les entreprises de terroir sont une capacité à savoir gérer la diversité dans de petites productions et à établir des liens avec des réseaux de distribution qui ne sont plus basés sur la massification de la consommation, mais sur la connexion de particularismes relativement substituables.

Rendre visible la qualité d'un produit devient un enjeu pour les industriels de l'agroalimentaire. La perception de la qualité ne peut être attachée, comme c'était le cas dans des marchés locaux, à la réputation fondée sur une connaissance 
interpersonnelle. Le changement d'échelle de l'action passe par le recours aux dispositifs fondés sur la confiance impersonnelle (Karpik, 1996). La réputation fondée sur une histoire - de la région ou de l'entreprise - doit être liée à une marque pour être visible ou à d'autres dispositifs impliquant une façon de produire certifiée par une autorité de contrôle (cas des labels en France). L'incertitude quant à la qualité des marchandises rend le marché opaque. Le seul critère de décision « rationnel » du consommateur devient alors le prix. Les produits de qualité qui ne peuvent se différencier des autres ont tendance à disparaître, leur coût de production étant en général plus élevé. La mauvaise qualité chasse la bonne qualité et produit ainsi la dissolution du marché (Akerlof, 1970). Il faut alors trouver les moyens de singulariser un type de production pour la soustraire au déclin prévisible. Alors que la consommation de viande de bœuf chutait après la crise de la vache folle, le taux de croissance de la viande de bœuf certifiée était de $978 \%$ entre 1996 et 1997 en France. Le report de consommation sur d'autres viandes s'est fait essentiellement sur le poulet labellisé.

Les dispositifs de normalisation et de certification rendent plus transparent un marché existant, mais ils peuvent aussi être considérés comme des médiateurs (Hennion, 1993) permettant la constitution de nouveaux marchés : c'est par exemple le cas de l'agriculture biologique (Sylvander, 1997). La mention agriculture biologique (AB) a été reconnue en 1990 en France. Depuis qu'il existe un cahier des charges clair et des organismes certifiant l'application de ce cahier des charges, le marché de l'agriculture biologique, jusqu'alors confidentiel, n'a cessé d'augmenter. Son chiffre d'affaires, de 3 milliards en 1996, est passé à 12 milliards, en 1997, en France.

La consommation des produits de terroir augmente grâce à cette exigence de qualité. En effet, dans l'esprit des consommateurs, terroir et qualité sont liés. Maintenir la croissance de ce type de marché suppose de conserver ce lien. Les indications de provenance, AOC au niveau français, AOP, IGP au niveau européen, n'attestent pas (comme les labels, la certification de conformité ou la mention $\mathrm{AB}$ ) d'une qualité contrôlée mais d'une provenance. Si l'identification (Callon, 1991) de terroir à qualité semble acquise, elle peut être remise en question. La méfiance est plus facile à construire que la confiance. L'enjeu est important, car si, pour l'instant, ce type de production est très éloigné des habitudes des grands groupes, il est très possible que ces derniers s'y intéressent davantage, advenant la croissance de ce marché. Dans ce dernier cas, comment les PME pourraient-elles garder leur rente?

Certaines grandes entreprises essaient déjà de s'engouffrer dans la brèche « terroir - qualité ». Par exemple, Lustucru lance des Maultaschen d'Alsace, des ravioles du Royans de la Drôme, des ravioles à la brousse de Provence. Ces lancements visent à concurrencer les fabricants régionaux très présents sur ces 


\section{Label, certificats, AOC, AOP, IGP et les autres...}

Le panorama de la réglementation s'appliquant aux produits alimentaires en France est pour le moins complexe, confus et, pour tout dire, incompréhensible par une majorité de producteurs et de... consommateurs ! On ne peut que souhaiter une clarification. En attendant, essayons d'y voir clair...

- Le label rouge et les labels régionaux (environ 350) sont deux composantes du label agricole créé en 1960. Le label national dit « label rouge » a été mis au point en 1973 par le ministère de l'Agriculture. Il garantit la qualité supérieure d'un produit, résultat d'exigences sévères et contrôlées par des organismes certificateurs, à tous les stades de la production, de transformation et de commercialisation du produit aboutissant à une qualité gustative. Les labels régionaux sont régis par le même cahier des charges et répondent aux mêmes exigences de contrôle.

- L'agriculture biologique, que signe le logo $\mathrm{AB}$, correspond à un mode d'agriculture. Elle garantit qu'un aliment est issu d'un mode de production attentif à l'environnement, qui s'interdit l'utilisation de produits chimiques de synthèse et respecte le bien-être des animaux. Le chiffre d'affaires de ce secteur représente environ $0,1 \%$ du marché des produits alimentaires en France. Il est cependant en progression soutenue en raison de la sensibilisation croissante des consommateurs aux questions des méthodes de production, en relation avec la qualité sanitaire des produits et avec le respect de l'environnement. Il existe un décalage entre la demande qui augmente rapidement et l'offre locale, ce qui entraîne des flux d'importation.

- L'appellation d'origine contrôlée (AOC), apparue en 1935 dans le secteur des vins, s'est étendue aux produits laitiers et concerne, depuis 1990, l'ensemble des produits agricoles et des denrées alimentaires. Cette appellation est un signe d'origine et de méthode de fabrication, non de qualité organoleptique, attribuée en France par l'INAO (Institut national des appellations d'origine). La qualité est liée au terroir et à ses variations (une année trop pluvieuse, et le vin est moins bon). L'AOC identifie un produit typique et spécifique, son incrustation dans le terroir et le savoir-faire des hommes qui le produisent. Les AOC concernent environ 133000 exploitations, soit $12,3 \%$ des exploitations recensées en 1997 et environ 650 produits, principalement des vins et fromages.

- L'appellation d'origine protégée (AOP) est une protection communautaire qui indique un lien fort avec le terroir, de l'origine de la matière première jusqu'à l'élaboration du produit fini. La production, la transformation et l'élaboration doivent avoir lieu dans une aire géographique déterminée. Exemple de produit bénéficiant de l'AOP : le fromage laguiole, fabriquée à partir de vaches Aubrac ayant pâturé de juin à septembre en altitude et produit dans les laiteries de l'Aubrac.

Le conflit opposant les tenants des appellations d'origine (pays du sud de l'Union européenne) et ceux qui considèrent ce type de «marquage » comme une 
entrave à l'exercice de la libre concurrence (pays du Nord) semble s'atténuer si l'on en juge par le nombre important de dossiers d'AOP (appellation d'origine protégée) déposés par les producteurs agricoles au nord comme au sud de l'Europe.

- L'indication géographique de provenance (IGP) atteste du lien avec le terroir à un des stades, au moins de la production, de la transformation ou de l'élaboration, et du fait que le produit jouit d'une grande réputation. C'est le cas de viandes provenant d'animaux élevés sur un terroir. Exemples de produits en attente de l'IGP : le jambon de Bayonne et le foie gras de canard du Sud-Ouest (les canards doivent être nés, élevés et engraissés sur le terroir). Rien qu'en France, l'IGP (indication géographique protégée) a fait l'objet de 54 demandes pour 194 labels en 1995.

- La certification de conformité est un nouvel outil de certification mis en place en 1990 en France et qui atteste que le produit possède des qualités ou suit des règles de fabrication particulières, strictement contrôlées. C'est la garantie d'une qualité régulière et distincte du produit courant. Cette certification atteste la vérité de ce qui est inscrit sur l'étiquette du produit.

créneaux où les PME peuvent entrer avec des investissements modérés ${ }^{6}$. La stratégie de Lustucru consiste à remettre en cause l'équivalence entre terroir et qualité : les PME seraient certes capables de fabriquer des produits du terroir, mais seules de grandes entreprises pourraient garantir la qualité. «Ces lancements sont destinés à initier l'ensemble des consommateurs aux produits régionaux avec la caution de la qualité Lustucru. » Nous pensons que cette construction d'image, en dépit des moyens financiers considérables qui sont engagés, ne sera guère pérenne en raison de l'antinomie existant entre la grande firme industrielle et le concept de produit de terroir tel que nous l'avons défini.

Le principal problème des PME de terroir consiste à construire une rente qui ne peut s'appuyer sur aucun mécanisme d'appropriation : les recettes ne sont pas brevetables et puisqu'elles ne sont pas nouvelles, elles sont en général connues. Le secret n'est efficace que dans le cas de nouvelles formulations. On peut penser à la création d'un goodwill d'image. Dans le cas d'une évolution de la consommation de plus en plus axée sur la qualité, il faut que les PME de terroir rendent robuste l'identification «terroir - qualité ». Cela ne peut se faire que par la constitution de cahiers des charges très stricts qui constitueraient des barrières à l'entrée pour les grandes entreprises. Par exemple, l'obligation de produire dans de petites unités,

6. Une ligne de production coûte environ 6 millions de francs. 
avec des règles d'approvisionnement sur les matières premières, irait totalement à l'encontre des pratiques habituelles des grandes entreprises ou des grands groupes de l'agroalimentaire.

Le poids économique des produits à forte identité pouvait être estimé (de façon très approximative compte tenu de la pénurie des statistiques dans ce domaine), de la façon suivante en 1994 :

\section{TABLEAU 3}

\section{Les produits alimentaires à identité « territoriale » en France et en Europe}

\begin{tabular}{lll}
\hline Paramètre (1994) & France & Union européenne \\
\hline Chiffre d'affaires & 90 milliards FRF $*$ & 250 milliards FRF \\
Poids dans la consommation & $10,6 \%$ & $7,3 \%$ \\
Nombre d'agriculteurs & 180000 & \\
Nombre d'entreprises de transformation & 2000 & \\
Nombre de points de vente & 4500 & \\
\hline
\end{tabular}

* Dont $92 \%$ pour les AOC, $7 \%$ pour les labels rouge et régionaux, $1 \%$ pour le label AB.

Il s'agit donc, au total, d'un secteur fortement diversifié en raison du nombre de produits concernés et socialement significatif.

La nouvelle «économie spatiale » fournit les bases théoriques nécessaires à la compréhension du lien entre territoire et activité économique. Il apparaît que désormais les espaces géographiques se structurent en fonction de complémentarités économique et sociale, et sur des bases historiques pour constituer des zones d'activité économique cohérente, que ce soit sur un territoire fortement peuplé (conurbations métropolitaines comme Paris, Londres, Barcelone ou cités-États comme Singapour, Hong Kong) ou zones rurales délimitées en «pays » (en France : la Beauce, le Roussillon, etc.). Le concept «d'économie d'archipel» forgé par Veltz (1996) est, à cet égard, très représentatif. Selon ce concept, ces territoires constituent des acteurs économiques en concurrence sur un marché globalisé pour capter des ressources financières, humaines ou symboliques (informations), les transformer en produits et les commercialiser. La compétitivité de chacun des territoires va très largement résulter des choix stratégiques effectués par les différents acteurs, selon le modèle proposé par Porter (stratégie de coût ou stratégie de différenciation). Pour la différenciation, l'existence d'un patrimoine culturel constitue un atout de premier plan. La différenciation par le territoire d'origine confère également au producteur une bonne protection contre le risque de délocalisation. 
À partir de cette caractérisation des «produits de terroir», il devient possible de passer aux stratégies des « acteurs-producteurs », les entreprises, permettant de qualifier un « groupe stratégique ». On s'appuiera, pour cela, sur la notion de « création de valeur».

\section{La valorisation des produits de terroir}

Les spécialistes des sciences de gestion mettent l'accent sur la «création de valeur » comme élément déterminant de la performance de l'entreprise. Plus précisément, on peut identifier des « actifs stratégiques » qui contribuent à la création de valeur : ces actifs, matériels ou immatériels, sont au nombre de quatre :

- les activités, qui se manifestent à travers les méthodes de fabrication et les produits eux-mêmes ;

- les ressources mobilisées pour spécifier le produit;

- les capacités, qui se traduisent par une aptitude à combiner produits et procédés de fabrication (métier);

- les compétences distinctives, qui, dans un métier donné, font l'originalité de l'entreprise.

À partir d'une grille proposée par Marchesnay (1995) pour formaliser le concept de création de valeur, on peut préciser les caractéristiques suivantes pour l'entreprise de terroir (figure 2).

C'est tout d'abord une entreprise qui élabore des produits originaux, qualifiés plus haut de produits de haute qualité spécifique (HQS). Ceci implique la conception et le respect d'un cahier des charges très strict sur le produit et le processus d'élaboration (activité).

C'est ensuite l'utilisation d'une histoire locale et d'un terroir, donc par essence une trajectoire dans le temps, et d'un lieu géographique bien délimité (ressources).

Les deux critères précédents (espace de production délimité, existence d'une base historique) élèvent des barrières à l'entrée dans le groupe stratégique et devraient limiter la mobilité des autres firmes du secteur agroalimentaire, dès lors qu'elles sont engagées sur d'autres configurations (produits standardisés, production de masse, etc.). Par ailleurs, les barrières à la mobilité jouent dans les deux sens, à l'entrée comme à la sortie. Les logiques de volume sont peu compatibles avec la fragmentation en micro-organisations, en multiterritoires imposées par le concept de terroir. 
FIGURE 2

\section{La création de valeur de l'entreprise de terroir}

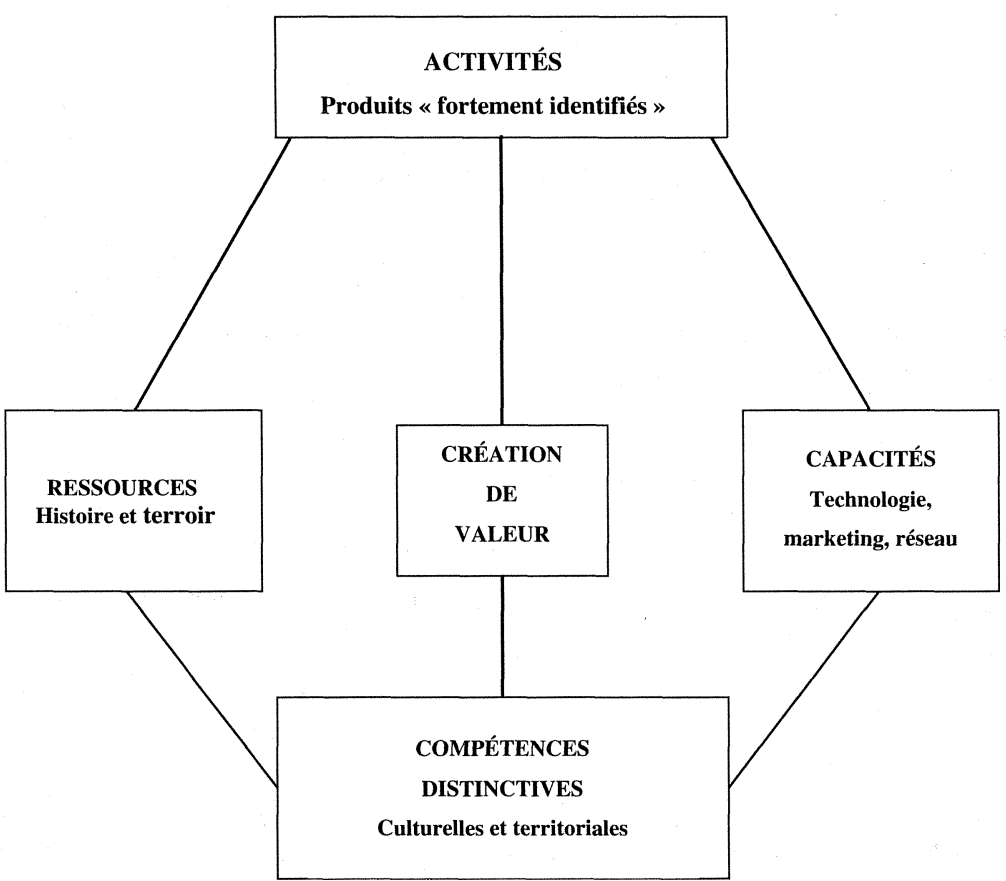

Source: Adapté d'un modèle propos par Marchesnay, 1995.

Le troisième «actif» est constitué des capacités propres du dirigeant et de son équipe : savoir-faire technique (fabrication selon une méthode traditionnelle, tout en respectant les exigences des normes réglementaires en matière d'hygiène, de sécurité et de respect de l'environnement) et mise en œuvre d'un marketing de haut niveau pour le produit (emballage, identification), mais aussi pour le contexte de la commercialisation (points de vente multiproduits à aménagement traditionnel intégré aux activités touristiques, «îlots » spécifiques en GMS, vente par correspondance, ce qui nécessite un bon ancrage dans des réseaux régionaux, nationaux et parfois internationaux de communication et de distribution). C'est là le domaine des capacités de l'entreprise.

Enfin, les compétences distinctives renvoient à la création d'une culture d'entreprise apte à combiner de façon durable activités / produits, ressources locales et capacités de production et de vente.

Ces conditions impliquent un profil très performant pour les responsables et les collaborateurs de ces «nouvelles entreprises de terroir» (NET) : compétences 


\section{Un exemple de nouvelles capacités managériales: le cyber-commerce}

La tendance actuelle est une concentration de la distribution. Il paraît assez peu probable de revenir à un éclatement de la distribution dont le cas extrême est celui des marchés locaux. La distribution virtuelle peut aussi se développer, puisque selon les dernières prévisions, les échanges commerciaux sur Internet pourraient être multipliés par 40 après l'an $2000^{1}$. Le cabinet d'études de marché anglais Datamonitor estime que les consommateurs européens dépenseront 3,2 milliards de dollars sur Internet en 2001 contre 96 millions de dollars en 1997. La population d'internautes estimée entre 40 et 65 millions au début de 1997 pourrait atteindre les 300 millions en l'an $2000^{2}$. Le marché est considérable et d'autant plus intéressant que les investissements nécessaires sont faibles (pas de loyer de magasin, d'entretien, de salaire du personnel, d'impôts locaux et autres taxes). Le stock peut même rester chez le producteur. Par contre, avec un tel type de commercialisation, les entreprises de transport vont jouer un rôle considérable. Il faut donc prendre en compte les coûts de transport et la qualité de ce dernier. Le métier et les compétences du cyber-commerçant restent pour l'instant à inventer.

Ainsi, le contexte de la mondialisation, loin de pénaliser les PME de terroir, leur offre au contraire deux types d'occasions favorables : faire connaître leurs produits en couplage avec les activités touristiques ; créer de nouveaux modes d'accès aux produits, grâce au « commerce électronique » (Internet).

1. Alberganti (1997).

2. Chiffres publiés par l'Union internationale des télécommunications (UIT, organisme des Nations Unies - Genève). Par ailleurs, une estimation récente indique que les ventes de produits alimentaires par catalogues Web devraient passer aux États-Unis de 500 millions de dollars en 1998 à 7 milliards en 2002, soit $18 \%$ des ventes électroniques totales, loin devant les livres ( 2 milliards).

techniques, bien sûr, mais aussi et surtout, car c'est le point faible actuellement, « intelligence-marketing » et aptitudes commerciales correspondant à la vente de produits haut de gamme. L'émergence d'une véritable « dynamique entrepreneuriale ${ }^{7}$ » est probablement le facteur décisif de la réussite des entreprises de terroir. En effet, on l'a compris, les NET doivent réaliser une difficile synthèse entre le patrimoine local, ses racines historiques et un marché de consommation alimentaire exigeant et mobilisant des techniques de commercialisation très avancées. On a donc un profil d'entreprises high tech, sur fond de produits à image traditionnelle. Il n'y a pas opposition entre terroir et innovation. Notre hypothèse est qu'en utilisant les deux

7. Sur les spécificités de la PME, voir Marchesnay (1995). 
leviers, confiance subjective (produit-tradition) et confiance-objective (technologies et management «dernier cri »), les PME de terroir adopteront un positionnement stratégique original susceptible de créer pour elles un «avantage concurrentiel » sur les marchés agroalimentaires.

\section{Le vivier des entreprises de terroir}

L'entreprise de terroir est nécessairement située dans les « franges » de l'oligopole des firmes dominantes de l'agroalimentaire représentées dans notre carte des groupes stratégiques. En effet, la grande firme (au-delà de 100 salariés) prend l'allure d'une unité industrielle, de production en grande quantité. L'entreprise de terroir doit conserver une « dimension humaine », marquée par la proximité entre les salariés. Elle appartiendra donc à la catégorie TPE (très petite entreprise, moins de 10 salariés) ou PE (petite entreprise, de 10 à 100 employés). Cependant, les capacités requises impliquent un minimum de division du travail et de qualification du personnel qui ne semble pas pouvoir exister en dessous de trois personnes à plein temps. Par ailleurs, les investissements nécessaires pour parvenir au niveau requis, tant dans le domaine technique que dans celui du marketing, conduisent également à un chiffre d'affaires (ou taille critique d'activité) minimal. Il est très difficile de proposer ici un indicateur tant la diversité des secteurs est grande dans l'agroalimentaire (ainsi un chiffre d'affaires d'un million de francs peut être atteint dans le vin avec 5000 bouteilles à $200 \mathrm{~F}$ s'il s'agit d'un grand cru classé du Bordelais ou 50000 cols à $20 \mathrm{~F}$ si l'on est en zone AOC du Languedoc). Il est sans doute préférable de raisonner à partir d'une analyse des coûts. Si l'on fait l'hypothèse d'un seuil d'investissement en communication à $100000 \mathrm{~F}$ par an, à raison d'un taux de $3 \%$ du chiffre d'affaires, on aboutit à des ventes de l'ordre de 3 millions de francs par an. Ce critère va écarter un grand nombre d'exploitations agricoles. Il faut enfin (ou plutôt au départ!), pour appartenir au groupe stratégique des PME de terroir, être en mesure de produire un PA-HQS et donc de posséder les actifs spécifiques décrits ci-dessus. Ces différents paramètres, au total très contraignants, conduisent à de faibles effectifs potentiels relatifs, si l'on considère la population totale d'entreprises de tout système alimentaire. On peut tenter, à partir des chiffres relatifs à l'industrie alimentaire européenne, une estimation.

Sur les 270000 entreprises de petite taille (moins de 100 salariés), on peut considérer qu'environ $10 \%$, soit de 25 à 30000 , possèdent les caractères requis pour évoluer vers le statut de PME de terroir. En termes d'emplois, cela représente un gisement considérable.

De nombreuses études, et en particulier les travaux du Commissariat Général du Plan présidé par Boissonnat sur «le travail dans 20 ans ${ }^{8}$, ont en effet montré

8. Boissonnat (1995). 
TABlEAU 4

Structure de l'industrie alimentaire UE-15 en 1992

\begin{tabular}{lllll}
\hline & $\begin{array}{l}\text { Nombre } \\
\text { d'entreprises }\end{array}$ & $\begin{array}{l}\text { Part du nombre } \\
\text { d'entreprises }\end{array}$ & $\begin{array}{l}\text { Part } \\
\text { de l'emploi }\end{array}$ & $\begin{array}{l}\text { Part } \\
\text { du CA }\end{array}$ \\
\hline$<20$ salariés & 256063 & $92,3 \%$ & $30,1 \%$ & $14,7 \%$ \\
20-99 salariés & 16545 & $6,0 \%$ & $18,1 \%$ & $15,8 \%$ \\
100 salariés et + & 4588 & $1,7 \%$ & $51,8 \%$ & $69,5 \%$ \\
277196 & $100,0 \%$ & 2,5 millions & 460 mds ECU & \\
\hline
\end{tabular}

Source : Nos calculs, d'après Commission européenne - DGIII, (1997), Panorama de l'industrie communautaire, OPOCE, Luxembourg, p. 13-5, 13-6.

que les petites entreprises de proximité étaient les seules dans nos économies contemporaines à créer des emplois. Ainsi, les entreprises de moins de 20 salariés représentent seulement $33 \%$ de la main-d'œuvre salariée en France, mais participent à la création de $95 \%$ des emplois depuis une dizaine d'années. Ces entreprises se trouvent principalement dans le secteur des services. Elles assurent aussi le maintien global de l'emploi dans les IAA (entreprises artisanales et PME) ${ }^{9}$ et contribuent à freiner son déclin dans l'agriculture (tourisme vert). À cet égard, les entreprises de terroir constituent, en faisant le lien entre la culture et l'alimentation, un atout significatif pour les régions rurales.

On doit néanmoins noter que la population des entreprises mentionnées ici présente un caractère extrêmement hétérogène en raison de la diversité des produits concernés et que le groupe stratégique des entreprises de terroir va trouver son fondement dans l'approche managériale au sein d'un système alimentaire complexe.

\section{Conclusion : Les conditions de l'émergence des PME de terroir}

Le groupe stratégique des PME de terroir est soumis, on vient de le voir, à de fortes contraintes du point de vue de la création de valeur: produits très différenciés, ressources technologiques et compétences marketing de haut niveau, bonnes capacités d'organisation en réseau en raison de leur taille réduite.

La stratégie des PME de terroir passe par l'utilisation et l'extension du réseau d'équivalence qui se met en place autour d'elles. Pour les consommateurs, qualité égale terroir ; pour les distributeurs, terroir égale produit d'appel ; pour les politiques,

9. Sur la période 1980-1994, les effectifs des IAA en France sont restés stables, alors que ceux de l'ensemble des industries de consommation courante ont chuté de $30 \%$. 
terroir égale aménagement du territoire. Le terroir commence à être au cœur d'un dispositif d'alliances intéressant. Pour préserver ces équations, il semblerait pertinent que les PME de terroir puissent s'entendre sur une stratégie commune afin de créer des barrières à l'entrée suffisamment élevées pour ne pas être balayées dès que le marché sera clairement ouvert. Il s'agit pour elles de rendre un réseau irréversible et de se doter d'un avantage comparatif très net en concevant des programmes d'action qui leur permettent à la fois d'assurer la qualité, la diversité et la mise en connexion transparente d'une offre et d'une demande éclatées.

Pour cela, il leur faudra peut-être mélanger des concepts statiques et dynamiques. La notion de terroir renvoie à une localisation géographique, à des traditions, à une culture. C'est une notion relativement statique. À l'opposé, le concept de traçabilité renvoie au cheminement d'un produit, à son déplacement et à sa transformation. Dans un cas, la confiance que le consommateur accorde à un produit est liée à la réputation subjective d'un territoire; dans l'autre, elle est liée à l'évaluation objectivée d'un parcours. En combinant les deux, les PME pourraient lier de façon forte et relativement irréversible la notion de terroir et celle de qualité.

Ce faisant, la notion de terroir peut aussi être fortement associée à celle d'innovation et même de haute technologie. L'innovation est le résultat d'un assemblage non habituel; elle peut consister à connecter des savoirs techniques et sociaux de différentes époques et de différentes zones géographiques. L'émergence des produits de terroir nous permet de comprendre pourquoi il est contre-productif d'associer ou de confondre évolution des techniques et innovation. En effet, la disparition de savoirs anciens peut diminuer la capacité d'innovation. Or, la notion de progrès technique suppose un sens «d'évolution-accumulation» des savoirs. En réalité, l'accumulation n'est pas possible. Il y a, en fait, substitution et donc disparition de certains savoirs et de certaines compétences. Les savoirs les plus facilement perdus sont ceux qui ne peuvent être codifiés : les connaissances tacites. Les produits du terroir peuvent donc être considérés comme des innovations prenant l'évolution des techniques à rebrousse-poil, car basées à la fois sur une tradition, une histoire, un savoir ancien récupéré et des techniques de production ou de commercialisation high tech. C'est, par exemple, dans une usine ultramoderne ${ }^{10}$ que Walkers produit les plus traditionnels des biscuits écossais : les shortbreads. C'est grâce à l'amélioration des procédés de transformation et de conditionnement que des productions de type apparemment artisanal peuvent plus facilement être diffusées. Jusqu'à présent, beaucoup d'innovations dans le secteur agroalimentaire étaient destinées à donner à des produits très standardisés une image de produits naturels ou traditionnels ${ }^{11}$. On peut imaginer que dans l'avenir certaines innovations

10. L'usine est située à Aberiour on Spey (Écosse).

11. Il en est ainsi, par exemple, de l'ajout d'arôme dans le Nescafé en poudre pour qu'il ait une odeur de café fraîchement moulu alors qu'en fait ce café en poudre n'a absolument aucune odeur. 
seront destinées à maintenir l'identité des produits traditionnels. Par exemple, en France, une PME a complètement robotisé sa production de «calissons d'Aix » afin de pouvoir combiner gestes traditionnels séculaires et augmentation de la productivité.

En résumé, sur le front pionnier des produits de terroir, deux comportements stratégiques sont envisageables. Le premier est déjà exploité par les très grandes firmes. Il tente, par des coûts massifs de communication (marque, emballage), de créer une image de « produits traditionnels » autour de produits standard. Le second pourrait l'être par des PME. Il s'agirait, par la mutualisation des coûts marketing ${ }^{12}$, au sein d'un territoire de convaincre les consommateurs que le produit de terroir est bien unique.

«Qualité, diversité, innovation, aménagement du territoire. » La stratégie des PME de terroir doit consister à favoriser et à maintenir ces mises en équivalence. Pour le faire, elles doivent réussir à combiner stratégie individuelle et stratégie collective, c'est-à-dire établir, au-delà de leurs intérêts individuels, un fonctionnement en réseau d'entreprises.

\section{Bibliographie}

AKERLOF, G.A. (1970), « The market for lemons : qualitative uncertainty and the market mechanism », Quarterly Journal of Economics, $\mathrm{n}^{\circ}$ 84, p. 488-500.

AKRICH, M., M. CALlON et B. LATOUR, (1988), «À quoi tient le succès des innovations. Deuxième épisode : L'art de choisir les bons porte-parole », Annales des Mines, septembre, p. 14-29.

Alberganti, M. (1997), «Internet, le grand supermarché», Le Monde de l'Économie, 16 septembre.

Boisard, P. et M.T. LeTABlier, (1987), « Le camembert : normand ou normé. Deux modèles de production dans l'industrie fromagère », Entreprises et Produits, Cahiers du Centre d'études pour l'emploi, Paris, Presses universitaires de France.

Boissonnat, J. (1995), Le travail dans vingt ans, Rapport du Commissariat Général du Plan, Paris.

Callon, M. (1991), «Réseaux technico-économique et irréversibilités », dans R. Boyer, B. Chavance et $\mathrm{O}$. Godard (dir.), Les figures de l'irréversibilité en économie, Paris, Éditions de l'École des Hautes Études en sciences sociales, p. 195-230.

CAUHAPÉ, V. (1998), «Les distributeurs de l'alimentaire utilisent les labels de qualité pour mieux vendre », Le Monde, 23 janvier, p. 25.

EYMARD-DUVERNAY, F. (1994), «Coordination des échanges par l'entreprise et qualité des biens », dans A. Orléan (dir.), Analyse économique des conventions, Paris, Presses universitaires de France, p. 307-334.

12. ... et l'utilisation de nouveaux vecteurs (Internet), sans pour autant négliger les incontournables réseaux classiques de proximité, dont font partie les GMS. 
HENNION, A. (1993), La passion musicale. Une sociologie de la médiation, Paris, Éditions Métailié.

Holmstrom, B.R. et J. Tirole, (1989) : «The theory of the firm», dans R. Schmalensee et R.D. Willings (éd.), Handbook of Industrial Organization, Elsevier Science Publishers B.V.

KARPIK, L. (1996), «Dispositifs de confiance et engagements crédibles », Sociologie du Travail, $\mathrm{n}^{\circ} 4$, p. 527-550.

MALASSIS, L. et G. GHERSI (1996), Économie agroalimentaire : concepts et méthodes. Traité d'économie agroalimentaire, Paris, Cujas.

MARCHESNAY, M. (1995), «La spécificité de la gestion des PME», exposé introductif au Colloque international sur les PME agroalimentaires, Université de Montpellier I/ CIRAD, 19-20 octobre, Montpellier.

MARChESNAY, M. (1997), «La convention, un outil de gestion?», Revue Française de Gestion, janvier-février, p. 114-123.

Porter, M. (1980), Competitive Strategy, New York, The Free Press, MacMillan Publishing Co., Traduction française (1982), Choix stratégiques et concurrence, Paris, Economica, p. 142-170.

Porter, M. (1985), Competitive Advantage : Creating and Sustaining Superior Performance, New York, The Free Press, MacMillan Publishing Co., Traduction française (1986), L'avantage concurrentiel : comment devancer ses concurrents et maintenir son avance, Paris, InterÉditions.

Porter, M. (1990), The Competitive Advantage of Nations, New York, The Free Press, MacMillan inc.

RASTOIN, J.L. (1992), «L'industrie alimentaire mondiale : vers un oligopole à franges », dans Politiques agricoles dans les PVD, ouvrage collectif sous la direction de M. Griffon, 3 tomes, numéro spécial de la revue Économie Politique, Paris, (1994), tome 2, p. 114-127.

RASTOIN, J.L. (1995), « Dynamique du système alimentaire français », Économie et Gestion Agroalimentaire, $\mathrm{n}^{\circ}$ 36, p. 5-14.

SYLVANDER, S. (1995), «Origine géographique et qualité des produits : approche économique », Revue du Droit Rural, $\mathrm{n}^{\circ} 237$, p. 465-473.

SYLVANDER, S. (1997), «Le rôle de la certification dans les changements de régime de coordination : l'agriculture biologique du réseau à l'industrie », Revue d'Économie Industrielle, $\mathrm{n}^{\mathrm{O}} 80,2^{\mathrm{e}}$ trimestre.

THÉVEnOt, L. (1986), «Les investissements de forme », Conventions Économiques, Paris, Presses universitaires de France.

Veltz, P. (1996), Mondialisation villes et territoires. L'économie d'archipel, Paris, Presses universitaires de France, 262 p.

VISSAC-CHARLES, V. (1996), «Caractérisation de la dynamique des réseaux : application à la gestion des projets d'innovation », dans C. Meadel et V. Rabeharisoa (dir.), Coordonner, Attribuer, Représenter, École des Mines de Paris, p. 297-305. 